Of the 153 patients enrolled, 78 were randomized to receive L-arginine and 75 to receive placebo. At 6 months, no significant difference between groups was found in left ventricular ejection fraction, vascular stiffness or plasma L-arginine levels. Six patients died in the L-arginine group, leading to early termination of the study because of safety concerns; five of the deaths were in patients aged 60 years or older.

The authors point out that vascular disease is associated with the expression of inducible nitric oxide synthase, leading to greatly increased nitric oxide levels. Excess nitric oxide contributes to vasodilatation and myocardial dysfunction. Exacerbation of these effects by L-arginine, together with age-related vascular dysfunction, might explain the deaths that occurred in the study. The authors conclude that L-arginine is unsuitable for patients following myocardial infarction, as it confers no improvement in cardiac function and might increase postinfarction mortality.

Original article Schulman SP et al. (2006) L-arginine therapy in acute myocardial infarction: the Vascular Interaction with Age in Myocardial Infarction (VINTAGE MI) randomized clinical trial. JAMA 295: 58-64

\section{Preprocedural clopidogrel increases bleeding in off-pump CABG}

Clopidogrel in combination with aspirin reduces the risk of stent thrombosis in percutaneous coronary revascularization procedures. Many patients with acute coronary syndromes are, therefore, given clopidogrel to provide prophylactic antiplatelet therapy before they undergo diagnostic angiography. The platelet inhibitory effects of preprocedural clopidogrel, however, are irreversible and can lead to bleeding complications and high re-exploration rates in cases where surgical revascularization (CABG) is needed. Off-pump CABG (OPCABG) has a lower risk of postoperative bleeding complications and subsequent need for re-exploration than conventional CABG. Researchers in the US have investigated whether preoperative clopidogrel reduces these advantages of OPCABG.

The study included 1,572 patients who underwent OPCABG (281 who received preoperative clopidogrel and 1,291 who did not; all patients received aspirin before surgery). Compared with patients not receiving clopidogrel, those receiving clopidogrel treatment were 5.1 times more likely to need re-exploration to control hemorrhage and 2.6 times more likely to receive a perioperative blood transfusion. Preoperative clopidogrel was not shown to be a significant predictor of operative mortality, but a larger sample size would be necessary to demonstrate such an effect.

The authors conclude that preoperative clopidogrel therapy has a detrimental effect on the beneficial antihemorrhagic effect of OPCABG. They suggest that clopidogrel should not be administered until it has been determined whether or not a patient is suitable for percutaneous coronary intervention.

Original article Kapetanakis EI et al. (2006) Effect of clopidogrel premedication in off-pump cardiac surgery: are we forfeiting the benefits of reduced hemorrhagic sequelae? Circulation 113: 1667-1674

\section{Kidney function level can be used to predict survival among patients with CVD}

Cardiovascular disease (CVD) is the major cause of morbidity and mortality in patients with chronic kidney disease. Researchers in Canada have explored the association between kidney function and survival in 8,521 patients who underwent cardiac catheterization, and have investigated the threshold at which risk of mortality becomes raised.

Patients were grouped into five broad kidney function categories based on their estimated glomerular filtration rates (eGFRs; $\geq 90 \mathrm{ml} / \mathrm{min} / 1.73 \mathrm{~m}^{2}, \quad 60-89 \mathrm{ml} / \mathrm{min} / 1.73 \mathrm{~m}^{2}$, $30-59 \mathrm{ml} / \mathrm{min} / 1.73 \mathrm{~m}^{2}, \quad<30 \mathrm{ml} / \mathrm{min} / 1.73 \mathrm{~m}^{2}$, and dialysis-dependent). The prevalence of CVD risk factors, such as peripheral vascular disease, cerebrovascular disease, congestive heart failure and hypertension (but not known hyperlipidemia), increased with decreasing kidney function. Survival over 4 years after cardiac catheterization decreased with decreasing kidney function, even after adjustment for severity of underlying CVD and clinical risk factors. Analysis of narrower $10 \mathrm{ml} / \mathrm{min} / 1.73 \mathrm{~m}^{2}$ eGFR categories showed similar decreases in survival with declining kidney function. When eGFR was modeled as a continuous variable, an increased risk of death was noted at an eGFR below $79 \mathrm{ml} / \mathrm{min} / 1.73 \mathrm{~m}^{2}$. A dose-response relationship was observed, with each $10 \mathrm{ml} / \mathrm{min} / 1.73 \mathrm{~m}^{2}$ decrease in eGFR below $70 \mathrm{ml} / \mathrm{min} / 1.73 \mathrm{~m}^{2}$ 